\title{
Transperitoneal vs retroperitoneal minimally invasive partial nephrectomy: comparison of perioperative outcomes and functional follow-up in a large multi-institutional cohort (The RECORD 2 Project)
}

\author{
Francesco Porpiglia ${ }^{1}$ - Andrea Mari ${ }^{2}$. Daniele Amparore ${ }^{1}$. Cristian Fiori ${ }^{1}$ - Alessandro Antonelli ${ }^{3}$. Walter Artibani ${ }^{4}$. \\ Pierluigi Bove ${ }^{5}$. Eugenio Brunocilla ${ }^{6,7}$. Umberto Capitanio ${ }^{8}$. Luigi Da Pozzo ${ }^{9}$. Fabrizio Di Maida ${ }^{1}$ Paolo Gontero ${ }^{10}$. \\ Nicola Longo ${ }^{11}$. Giancarlo Marra ${ }^{10}$. Bernardo Rocco ${ }^{12,13} \cdot$ Riccardo Schiavina $^{6}$. Claudio Simeone ${ }^{3}$. \\ Salvatore Siracusano ${ }^{4} \cdot$ Riccardo Tellini $^{1} \cdot$ Carlo Terrone $^{14}$. Donata Villari ${ }^{15} \cdot$ Vincenzo Ficarra $^{16} \cdot$ Marco Carini $^{1}$. \\ Andrea Minervini ${ }^{1,17}$ (1) The RECORD 2 Project
}

Received: 6 March 2020 / Accepted: 17 August 2020 / Published online: 27 August 2020

(c) The Author(s) 2020

\begin{abstract}
Background Aim of this study was to evaluate and compare perioperative outcomes of transperitoneal (TP) and retroperitoneal (TR) approaches in a multi-institutional cohort of minimally invasive partial nephrectomy (MI-PN).

Material and methods All consecutive patients undergone MI-PN for clinical T1 renal tumors at 26 Italian centers (RECORd2 project) between 01/2013 and 12/2016 were evaluated, collecting the pre-, intra-, and postoperative data. The patients were then stratified according to the surgical approach, TP or RP. A 1:1 propensity score (PS) matching was performed to obtain homogeneous cohorts, considering the age, gender, baseline eGFR, surgical indication, clinical diameter, and PADUA score. Results 1669 patients treated with MI-PN were included in the study, 1256 and 413 undergoing TP and RP, respectively. After 1:1 PS matching according to the surgical access, 413 patients were selected from TP group to be compared with the 413 RP patients. Concerning intraoperative variables, no differences were found between the two groups in terms of surgical approach (lap/robot), extirpative technique (enucleation vs standard PN), hilar clamping, and ischemia time. Conversely, the TP group recorded a shorter median operative time in comparison with the RP group (115 vs 150 min), with a higher occurrence of intraoperative overall, 21 (5.0\%) vs 9 (2.1\%); $p=0.03$, and surgical complications, 18 (4.3\%) vs 7 (1.7\%); $p=0.04$. Concerning postoperative variables, the two groups resulted comparable in terms of complications, positive surgical margins and renal function, even if the RP group recorded a shorter median drainage duration and hospital length of stay (3 vs 2 for both variables), $p<0.0001$.

Conclusions The results of this study suggest that both TP and RP are feasible approaches when performing MI-PN, irrespectively from tumor location or surgical complexity. Notwithstanding longer operative times, RP seems to have a slighter intraoperative complication rate with earlier postoperative recovery when compared with TP.
\end{abstract}

Keywords Renal cell carcinoma $\cdot$ Minimally invasive partial nephrectomy $\cdot$ Transperitoneal $\cdot$ Retroperitoneal $\cdot$ Surgical approach

The members of The RECORD 2 Project have been listed in acknowledgements.

Electronic supplementary material The online version of this article (https://doi.org/10.1007/s00464-020-07919-4) contains supplementary material, which is available to authorized users.

Andrea Minervini

andreamine@libero.it

Extended author information available on the last page of the article
The incidental detection of renal masses increased over the last decades due to the expanding use of imaging diagnosis performed for other reasons [1]. Alongside with this occurrence, kidney surgery has undergone both theoretical and technical developments [2, 3]. Firstly, the indication for partial nephrectomy $(\mathrm{PN})$ in elective cases has been expanded widely, being well demonstrated the advantages of partial over radical surgery in $\mathrm{T} 1$ tumors in terms of functional preservation with similar oncological safety [4-6]. On the other hand, the technological improvements of the last 30 
years paved the way for the gradually increased employment of minimally invasive approaches, first with the advent of laparoscopy and then with robotics, progressively minimizing the impact and invasiveness of surgical procedures [7-9].

The surgical access, either trans or retroperitoneal (TP or RP), plays a key role in kidney surgery, since it meaningfully influences the comfort of the procedure as well as perioperative outcomes $[10,11]$. To date, the choice between $\mathrm{TP}$ or RP access has been traditionally influenced by tumor(location and surgical complexity) and patient-related features (previous abdominal surgery, presence of abundant abdominal fat) $[12,13]$. However, in the last few years, the ongoing improvements of computer-enhanced technology have provided the necessary means for surgeons to assimilate minimally invasive surgical options into their everyday surgical practice and overcome the surgical limitation related to conventional surgery. In this light, robotic surgery made easier to perform even complex surgical procedures with both accesses thanks to an improved ergonomic position for the surgeon, three-dimensional vision of the operating field and augmented maneuverability of the instruments due the EndoWrist $®$ technology.[14].

Nevertheless, to date, the final choice between TP or RP approach still remains strictly influenced by the surgeon preference and the advantages of different surgical access during laparoscopic or robotic PN has not been clearly highlighted yet $[15,16]$.

To fill this gap, the aim of the current study was to evaluate and compare the perioperative outcomes in patients treated with TP and RP minimally invasive PN for clinical T1 renal tumors in a large multi-institutional study (The RECORD 2 project).

\section{Materials and methods}

The Italian REgistry of COnservative and Radical Surgery for cortical renal tumor Disease (RECORD 2 Project) is a prospective observational multicenter project promoted by the Italian Society of Urology (SIU). This study was approved by the local ethics committee, and written informed consent was collected for all the patients. Overall 4325 consecutive patients undergone renal surgery for cortical renal tumors at 26 urological Italian centers between January 1, 2013, and December 31, 2016, were included. For each patient, anthropometric and preoperative data, imaging, indications and co-morbidities, intraoperative data, postoperative data, histological analysis, follow-up were collected in an e-form central database to limit missing or wrong data inputs. Comorbidity status was evaluated by Charlson comorbidity index (CCI), physical status (PS) by the American Society of Anesthesiologists (ASA) classification system. Surgical indications were defined as elective (unilateral lesion with healthy contralateral kidney), relative (presence of diabetes, hypertension or lithiasis that could potentially affect kidney function in the future) and absolute (bilateral tumors, multiple tumors, moderate to severe CKD or tumors involving an anatomically or functionally solitary kidney). Center experience was evaluated as number of PN/ year and PN/RN ratio per year. Surgical approach and type of resection were chosen according to surgeons' preference and centers availability.

For the purpose of this study, only patients undergone minimally invasive PN (mi-PN) for clinical T1 renal tumors were included at a first stage. Therefore, 1712 patients treated with radical nephrectomy and 886 patients treated with open PN, 47 patients with $\mathrm{cT} \geq 2$ and 11 patients with missing data undergone minimally invasive PN were excluded and only patients undergone minimally invasive TP $(n=1256)$ and RP $(n=413)$ from 21 centers were included.

\section{Statistical analysis}

A propensity score matching was performed on the selected cohort to settle on two cohorts of patients undergone TP and RP MI-PN with comparable preoperative clinical features [17]. The matching was carried out with a 1:1 ratio with respect to the surgical access (413 TP vs $413 \mathrm{RP}$ mi-PN) with a $\mathrm{C}$ statistic of 0.67 adjusting for the variables: age, gender, baseline eGFR, surgical indication, clinical diameter, ECOG and ASA PS scores, tumor location and PADUA score. The difference between the two groups is plotted in Supplementary Fig. 1. The Student t-test and the Mann-Whitney- $U$ test were used to compare continuous to categorical variables and the Pearson's Chi-square test was used to compare two categorical variables. Statistical significance was set at $p<0.05$. All reported $p$ values were two-sided. Analyses were carried out with RStudio graphical interface v.0.98 for R software environment v.3.0.2, using the packages MatchIt, rms and histbackback, and with STATA v.14.1 (StataCorp LP, College Station, TX).

\section{Results}

\section{Patients and tumors features}

Preoperative tumor and patient features of the selected cohorts after propensity score matching are reported in Table 1. After propensity score matching, all the variables were comparable between the two groups. In detail, patients included in the TP and RP cohorts were males in $65.6 \%$ and $61.8 \%$ of the cases $(p=0.67)$, respectively. Patients had a median (interquartile range [IQR]) age of 64.8 (56.2-72.4) and $64.6(55.9-72.3)$ years $(p=0.71)$, and a median (IQR) BMI of 25.9 (23.9-28.7) and $25.8(23.7-28.4)$ in the TP 
and RP group $(p=0.95)$, respectively. The CCI score and the ASA PS score were $1(0-2)$ and $2(2-3)$ for both groups. Patients had relative and absolute indication for surgery in $20.8 \%$ and $3.7 \%$ of TP group versus $24.9 \%$ and $5.5 \%$ of the $\mathrm{RP}$ group $(p=0.24)$.

A clinical T1a (cT1a) and cT1b stage were reported in $79.9 \%$ and $20.1 \%$ of TP while in $80.1 \%$ and $19.9 \%$ of the RP patients $(p=0.82)$, respectively. The median (IQR) PADUA score was 7 (7-8) for both groups $(p=0.69)$, with PADUA $\geq 10$ registered in $3.4 \%$ of TP and $3.9 \%$ of RP patients, respectively. The propensity score matching evened the differences also in terms of tumor location: the percentage of tumors located on the lateral margin, medial margin, anterior face and posterior face were $33.7 \%$ vs $34.6 \%, 11.8 \%$ vs $11.3 \%, 9.9 \%$ vs $9.0 \%$ and $44.3 \%$ vs $45.0 \%$ for TP and RP groups, not considering the surgical access. At baseline the median (IQR) values of preoperative creatinine were $0.9 \mathrm{mg} /$ $\mathrm{dl}(0.8-1)$ and $0.9 \mathrm{mg} / \mathrm{dl}(0.8-1.1)$, with an eGFR of $85.1 \mathrm{ml} /$ $\mathrm{min} / \mathrm{m}^{2}(70.3-96.6)$ and $82.8 \mathrm{ml} / \mathrm{min} / \mathrm{m}^{2}(66.8-99.5)$ for TP and RP cohort, respectively.

\section{Surgical features}

The surgical features of the two cohorts after propensity score matching are summarized in Table 2.

Overall, 12/21 (57.1\%) and 6/21 (28.6\%) centers performed $>30$ and $>50$ mi-PNs per year, respectively. An overview of the distribution of surgical accesses for each center is shown in Fig. 1. Patients undergone RP were treated in centers performing a median (IQR) of 71 (44-84) PNs/year, while the matched patients undergone TP underwent surgery in centers with a median of $56(35-79) \mathrm{PN} / \mathrm{year}$ $(p<0.0001)$. Specifically, in the RP cohort, $331(80.2 \%)$ and $315(76.3 \%)$ patients were treated in centers performing $>30$ and $>50$ PNs/year, while of the TP cohort only 313 (75.8\%) and $256(62.0 \%)$ patients underwent surgery in centers performing $>30$ and $>50$ PNs/year $(p<0.001)$. Moreover, the median PN/RN ratio was $74.0 \%(66.6-87.9 \%)$ and $66.6 \%$ (50.6-78\%) for RP and TP groups, respectively $(p<0.001)$.

Laparoscopic PN (LPN) and robot-assisted PN (RAPN) were planned in $281(68.0 \%)$ and $132(32.0 \%)$ cases with TP access versus $279(67.5 \%)$ and $134(33.5 \%)$ cases with RP access, respectively $(p=0.67)$.

Considering the hilar clamping approach, in $47.9 \%$ of the TP and $51.6 \%$ of the RP surgeries, renal pedicle was not clamped. When hilar clamping was performed, the median (IQR) ischemia time was 19 (14-24) and $20(15-25) \mathrm{min}$ for the two groups $(p=0.14)$. Simple enucleation was performed in $41.9 \%$ of the TP and $40.9 \%$ of the RP, respectively $(p=0.77)$.

Median EBL for the two groups were similar $(100 \mathrm{ml}$ and $150 \mathrm{ml}, p=0.10$ ). Conversely, the RP group had shorter median intraoperative (skin to skin) time compared the TP group (115 vs $150 \mathrm{~min}, p<0.0001)$.

The intraoperative overall complications rate $(5 \%$ vs $2.1 \%, p=0.03)$ and the intraoperative surgical complications rate $(4.3 \%$ vs $1.7 \%, p=0.04)$ were higher in the TP compared to the RP group. Conversely, no differences between

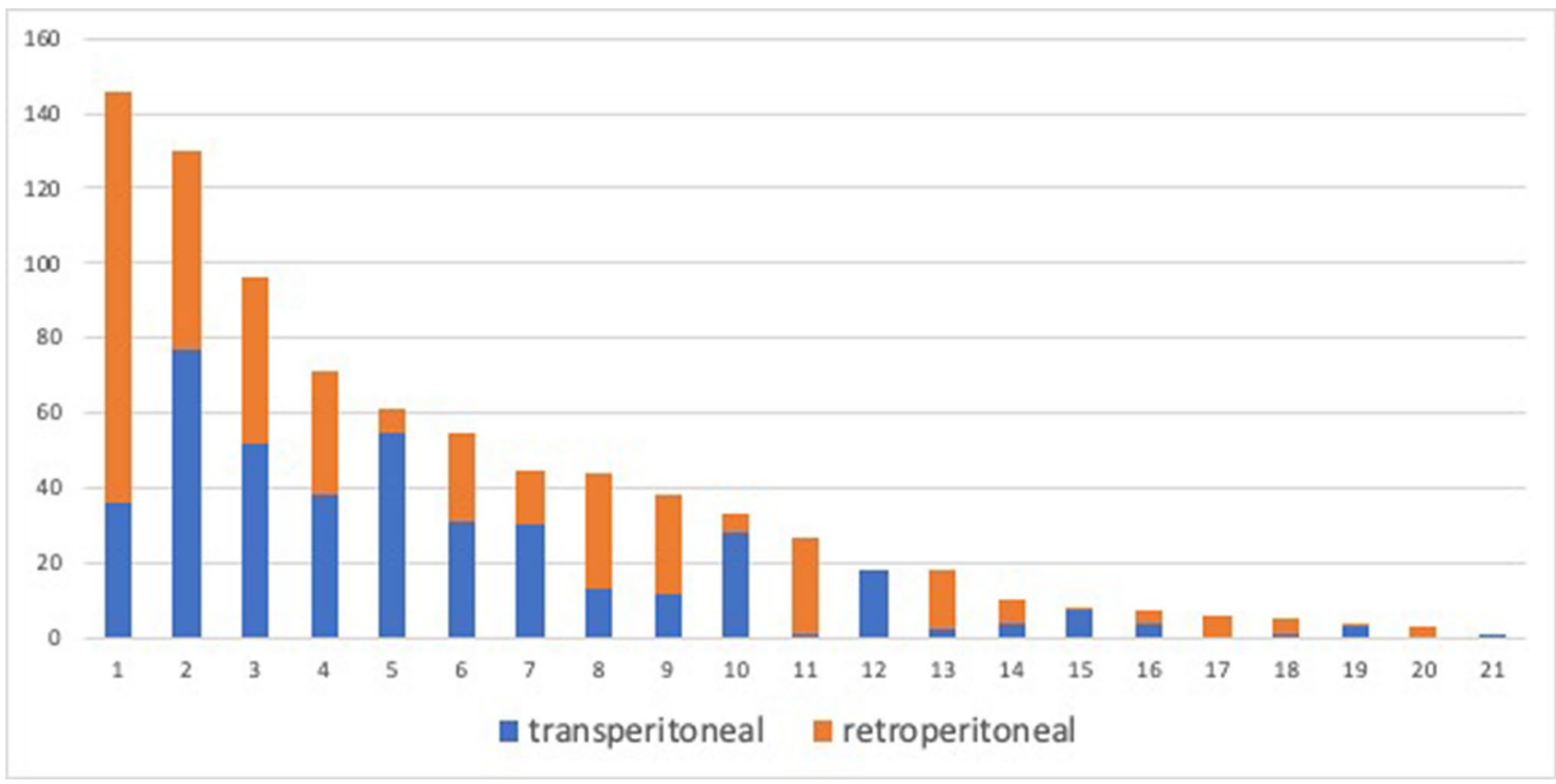

Fig. 1 Distribution of transperitoneal and retroperitoneal surgical procedures according to each single centre after propensity score matching analysis 
Table 1 Comparison of the preoperative characteristics of 413 patients treated with retroperitoneal minimally invasive partial nephrectomy with selected matched 413 patients treated with transperitoneal minimally invasive partial nephrectomy for renal tumors within the RECORD 2 project

\begin{tabular}{|c|c|c|c|c|c|}
\hline \multirow{2}{*}{$\begin{array}{l}\text { RECORD } 2 \text { project-preoperative tumor and } \\
\text { patient data }\end{array}$} & \multicolumn{4}{|c|}{ Surgical access } & \multirow[t]{2}{*}{$p$ value } \\
\hline & \multicolumn{2}{|c|}{$\begin{array}{l}\text { Transpertioneal } \\
(n=413)\end{array}$} & \multicolumn{2}{|c|}{$\begin{array}{l}\text { Retroperitoneal } \\
(n=413)\end{array}$} & \\
\hline \multicolumn{6}{|l|}{ Gender, $n . \%$} \\
\hline Male & 271 & $65.6 \%$ & 280 & $67.8 \%$ & 0.67 \\
\hline Female & 142 & $34.4 \%$ & 133 & $32.2 \%$ & \\
\hline Age (years), median IQR & 64.8 & $56.2-72.4$ & 64.6 & $55.9-72.3$ & 0.71 \\
\hline BMI, median IQR & 25.9 & $23.9-28.7$ & 25.8 & $23.7-28.4$ & 0.95 \\
\hline \multicolumn{6}{|l|}{ ECOG score, $n . \%$} \\
\hline 0 & 311 & $75.3 \%$ & 315 & $76.3 \%$ & 0.69 \\
\hline$\geq 1$ & 92 & $24.6 \%$ & 98 & $23.7 \%$ & \\
\hline CCI score, median IQR & 1 & $0-2$ & 1 & $0-2$ & 0.71 \\
\hline ASA PS score, median IQR & 2 & $2-3$ & 2 & $2-3$ & 0.82 \\
\hline \multicolumn{6}{|l|}{ Indication, $n . \%$} \\
\hline Elective & 312 & $75.5 \%$ & 287 & $69.6 \%$ & 0.24 \\
\hline Relative & 86 & $20.8 \%$ & 103 & $24.9 \%$ & \\
\hline Absolute & 19 & $3.7 \%$ & 23 & $5.5 \%$ & \\
\hline \multicolumn{6}{|l|}{ Clinical T, $n . \%$} \\
\hline $\mathrm{T} 1 \mathrm{a}$ & 330 & $79.9 \%$ & 331 & $80.1 \%$ & 0.82 \\
\hline $\mathrm{T} 1 \mathrm{~b}$ & 83 & $20.1 \%$ & 82 & $19.9 \%$ & \\
\hline \multicolumn{6}{|l|}{ Tumor side, $n . \%$} \\
\hline Right & 202 & $48.9 \%$ & 194 & $47.0 \%$ & 0.93 \\
\hline Left & 211 & $51.1 \%$ & 219 & $53.0 \%$ & \\
\hline Clinical diameter, median IQR & 3.2 & $1.9-4.6$ & 3.1 & $1.8-4.5$ & 0.63 \\
\hline \multicolumn{6}{|l|}{ Tumor site, $n . \%$} \\
\hline Mesorenal & 271 & $65.6 \%$ & 278 & $67.3 \%$ & 0.18 \\
\hline Polar & 142 & $34.4 \%$ & 135 & $32.7 \%$ & \\
\hline \multicolumn{6}{|l|}{ Tumor growth pattern, $n . \%$} \\
\hline$\geq 50 \%$ Exophytic & 229 & $55.4 \%$ & 240 & $58.1 \%$ & 0.41 \\
\hline$<50 \%$ Exophytic & 160 & $38.7 \%$ & 147 & $35.6 \%$ & \\
\hline Entirely endophytic & 24 & $5.8 \%$ & 26 & $6.3 \%$ & \\
\hline PADUA Score, median IQR & 7.0 & $7.0-8.0$ & 7.0 & $7.0-8.0$ & 0.69 \\
\hline \multicolumn{6}{|l|}{ PADUA complexity group, n. $\%$} \\
\hline Low $(6-7)$ & 244 & $59.1 \%$ & 251 & $60.8 \%$ & 0.78 \\
\hline Medium (8-9) & 155 & $37.5 \%$ & 146 & $35.4 \%$ & \\
\hline $\operatorname{High}(\geq 10)$ & 14 & $3.4 \%$ & 16 & $3.9 \%$ & \\
\hline \multicolumn{6}{|l|}{ Tumor localization, $n . \%$} \\
\hline Lateral margin & 139 & $33.7 \%$ & 143 & $34.6 \%$ & 0.26 \\
\hline Medial margin & 49 & $11.8 \%$ & 46 & $11.3 \%$ & \\
\hline Anterior face & 41 & $9.9 \%$ & 37 & $9.0 \%$ & \\
\hline Posterior face & 183 & $44.3 \%$ & 187 & $45.0 \%$ & \\
\hline Peri-hilar & 1 & $0.2 \%$ & 0 & $0 \%$ & \\
\hline Preoperative hemoglobin, mean SD & 14.5 & $13.4-15.3$ & 14.2 & $13.3-15.2$ & 0.83 \\
\hline Preoperative creatinine $(\mathrm{mg} / \mathrm{dl})$, mean SD & 0.9 & $0.80-1.00$ & 0.9 & $0.80-1.10$ & 0.19 \\
\hline Preoperative eGFR, median IQR & 85.1 & $70.3-96.6$ & 82.8 & $66.8-99.5$ & 0.67 \\
\hline
\end{tabular}

the groups were recorded in terms of intraoperative medical complications $(0.7 \%$ vs $0.5 \%, p=0.64)$.

\section{Postoperative and functional features}

Postoperative and functional outcomes of the two cohorts after propensity score matching are shown in Table 3. No differences were found in terms of postoperative complication 
Table 2 Comparison of the centers data and the intraoperative characteristics of 413 patients treated with retroperitoneal minimally invasive partial nephrectomy with selected matched 413 patients treated

\begin{tabular}{|c|c|c|c|}
\hline \multirow[t]{2}{*}{ RECORD 2 project-intraoperative center and surgical data } & \multicolumn{2}{|l|}{ Surgical access } & \multirow[t]{2}{*}{$p$ value } \\
\hline & Transpertioneal $(n=413)$ & Retroperitoneal $(n=413)$ & \\
\hline PN/RN ratio, median (IQR) & $66.6 \%(50.6-78 \%)$ & $74.0 \%(66.6-87.9 \%)$ & $<0.0001$ \\
\hline Number of PN/year of the center of treatment, median (IQR) & $56(35-79)$ & $71(44-84)$ & $<0.0001$ \\
\hline \multicolumn{4}{|l|}{ Center volume, $n(\%)$} \\
\hline$>30 \mathrm{PN} /$ year & $313(75.8 \%)$ & $331(80.1 \%)$ & 0.001 \\
\hline$>50 \mathrm{PN} /$ year & $256(62.0 \%)$ & $315(76.3 \%)$ & 0.001 \\
\hline \multicolumn{4}{|l|}{ Surgical approach } \\
\hline Laparoscopic & $281(68.0 \%)$ & $279(67.5 \%)$ & \multirow[t]{2}{*}{0.67} \\
\hline Robotic & $132(32.0 \%)$ & $134(33.5 \%)$ & \\
\hline \multicolumn{4}{|l|}{ Technique, $n(\%)$} \\
\hline Enucleation & $173(41.9 \%)$ & $169(40.9 \%)$ & \multirow[t]{2}{*}{0.77} \\
\hline Standard PN & $240(58.1 \%)$ & $244(59.1 \%)$ & \\
\hline \multicolumn{4}{|l|}{ Hilar clamping, $n(\%)$} \\
\hline Not performed & $198(47.9 \%)$ & $213(51.6 \%)$ & \multirow[t]{2}{*}{0.29} \\
\hline Performed & $215(52.1 \%)$ & $200(48.4 \%)$ & \\
\hline Ischemia time(min), median (IQR) & $19(14-24)$ & $20(15-25)$ & 0.14 \\
\hline EBL (cc), median (IQR) & $100(100-200)$ & $150(100-143)$ & 0.10 \\
\hline Extended peritoneum opening, $n(\%)$ & $32(7.7 \%)$ & - & - \\
\hline Intraoperative time, median (IQR) & $115(96-130)$ & $150(120-180)$ & $<0.0001$ \\
\hline Intraoperative overall complications, $n(\%)$ & $21(5.0 \%)$ & $9(2.1 \%)$ & 0.03 \\
\hline Intraoperative surgical complications, $n(\%)$ & $18(4.3 \%)$ & $7(1.7 \%)$ & 0.04 \\
\hline Vascular lesion, $n(\%)$ & $9(2.1 \%)$ & $4(0.8 \%)$ & - \\
\hline Spleen injury, $n(\%)$ & $2(0.4 \%)$ & - & - \\
\hline Conversion to open procedure, $n(\%)$ & $4(0,8 \%)$ & $2(0.4 \%)$ & - \\
\hline Bleeding from renal resection bed, $n(\%)$ & $3(0.2 \%)$ & $1(0.2 \%)$ & - \\
\hline Intraoperative medical complications, $n(\%)$ & $3(0.7 \%)$ & $2(0.5 \%)$ & 0.64 \\
\hline Miocardial infarction, $n(\%)$ & $2(0.4 \%)$ & $1(0.2 \%)$ & - \\
\hline Arrytmias, $n(\%)$ & $1(0.2 \%)$ & $1(0.2 \%)$ & - \\
\hline
\end{tabular}

with transperitoneal minimally invasive partial nephrectomy for renal tumors within the RECORD 2 project 
Table 3 Comparison of the postoperative outcomes of 413 patients treated with retroperitoneal minimally invasive partial nephrectomy with selected matched 413 patients treated with transperitoneal minimally invasive partial nephrectomy for renal tumors within the RECORD 2 project

\begin{tabular}{|c|c|c|c|}
\hline \multirow{2}{*}{$\begin{array}{l}\text { RECORD } 2 \text { project—report of early postoperative and functional follow-up } \\
\text { outcomes }\end{array}$} & \multicolumn{2}{|l|}{ Surgical access } & \multirow[t]{2}{*}{$p$ value } \\
\hline & Transpertioneal $(n=413)$ & Retroperitoneal $(n=413)$ & \\
\hline Surgical postoperative complications, $n(\%)$ & $29(7 \%)$ & $25(6.1 \%)$ & 0.57 \\
\hline Surgical clavien 2 postop complications, $n(\%)$ & $12(2.9 \%)$ & $11(2.7 \%)$ & \\
\hline Surgical clavien $3 \mathrm{a}$, postop complications, $n(\%)$ & $11(2.7 \%)$ & $10(2.4 \%)$ & \\
\hline Surgical clavien $3 \mathrm{~b}$, postop complications, $n(\%)$ & $3(0.7 \%)$ & $4(1 \%)$ & \\
\hline Surgical clavien $4 \mathrm{a}$, postop complications, $n(\%)$ & $3(0.7 \%)$ & 0 & \\
\hline Transfusions, $n(\%)$ & $21(5.1 \%)$ & $15(3.6 \%)$ & \\
\hline Deep vein thrombosis, $n(\%)$ & $1(0.2 \%)$ & 0 & \\
\hline Superselective embolization, $n(\%)$ & $8(2.0 \%)$ & $7(1.7 \%)$ & \\
\hline Urinary fistula treated without stenting, $n(\%)$ & $4(0.8 \%)$ & $4(0.8 \%)$ & \\
\hline Urinary fistula treated with stenting or nephrostomy tube, $n(\%)$ & $2(0.4 \%)$ & $1(0.2 \%)$ & \\
\hline Medical postoperative complications, $n(\%)$ & $16(3.9 \%)$ & $14(3.4 \%)$ & 0.48 \\
\hline Respiratory complications, $n(\%)$ & $8(2.0 \%)$ & $8(2.0 \%)$ & \\
\hline Cardiologic complications, $n(\%)$ & $7(1.7 \%)$ & $5(1.2 \%)$ & \\
\hline Acute pulmonary embolism, $n(\%)$ & $1(0.2 \%)$ & $1(0.2 \%)$ & \\
\hline Drainage duration (days), median (IQR) & $3(2-4)$ & $2(1-2)$ & $<0.0001$ \\
\hline Time to bowel canalization, median (IQR) & $3(2-5)$ & $2(1-3)$ & $<0.0001$ \\
\hline Hospital length of stay (days), median (IQR) & $3(3-4)$ & $2(2-3)$ & $<0.0001$ \\
\hline Malignant tumors, $n(\%)$ & $308(74.6 \%)$ & $310(75.1 \%)$ & 0.82 \\
\hline Positive surgical margins, $n(\%)$ & $21(5.1 \%)$ & $23(5.6 \%)$ & 0.73 \\
\hline Preoperative-1st postoperative day $\Delta$ hemoglobin (mg/dL), median (IQR) & $1.7(1.1-3.0)$ & $1.5(0.9-2.7)$ & 0.48 \\
\hline Preoperative-3rd postoperative day $\Delta$ hemoglobin (mg/dL), median (IQR) & $2.2(1.4-3.1)$ & $1.8(1.0-2.9)$ & 0.25 \\
\hline 1st POD creatinine (mg/dl), median (IQR) & $1.0(0.8-1.2)$ & $1.0(0.8-1.3)$ & 0.18 \\
\hline 1st POD eGFR, median (IQR) & $76(61.0-89.0)$ & $73(57.2-89.0)$ & 0.08 \\
\hline Preoperative-1st POD $\Delta$ eGFR, median (IQR) & $10.3(0.0-20.8)$ & $10.4(0.0-22.5)$ & 0.69 \\
\hline Preoperative-3rd POD $\Delta$ eGFR, median (IQR) & $10.1(0.0-20.1)$ & $9.0(0.0-20.7)$ & 0.53 \\
\hline Preoperative-6th month $\Delta$ eGFR, median (IQR) & $8.7(0.3-15.9)$ & $8.8(0.4-15.0)$ & 0.65 \\
\hline Preoperative-12th month $\Delta$ eGFR, median (IQR) & $9.2(4.1-21.5)$ & $9.3(3.4-20.8)$ & 0.67 \\
\hline Preoperative-24th month $\Delta$ eGFR, median (IQR) & $9.6(4.4-20.5)$ & $9.8(4.5-20.0)$ & 0.68 \\
\hline
\end{tabular}

The progressively greater ability of surgeons with minimally invasive approaches led to a significantly rising adoption of RP access [10-12, 15]. The RP access can be extremely challenging from a surgical standpoint due to (1) the limited working space which makes the development of RP space a meticulous and crucial step and (2) the possibility of breaching the peritoneum (3) a slightly harder identification and isolation of the anatomical landmarks. However, the RP access in mi-PN allows to gain a prompt and direct access to renal hilum to reduce operative time and the risk of renal pedicle injury during its isolation [15]. Furthermore, the constraint of the procedure in the retroperitoneum allows to minimize the postoperative ileus and even more significantly reduce the postoperative distress and facilitate an early discharge.

In this study, a large, nation-based and prospectively compiled contemporary dataset was analyzed to compare the perioperative and medium-term functional outcomes in patients with cT1 renal tumors and comparable characteristics at baseline, through a propensity score matching, and undergone TP versus RP mi-PN [21]. We found that RP mi-PN was more significantly performed in centers with higher caseload and a higher attitude to perform conservative surgery. Patients undergone RP mi-PN had a significantly lower rate of overall and surgical intraoperative complications, a lower time of drain maintenance and postoperative hospital stay compared to those patients treated with TP mi-PN. Conversely, intraoperative time was significantly lower in TP mi-PN procedures compared to their counterparts. Furthermore, our results showed comparable outcomes between RP and TP mi-PN in terms of pedicle clamping and ischemia time, postoperative complications, surgical margins, and short- and medium-term renal function recovery. 
Our study suggests that both accesses, either TP or RP, are generally feasible and allow comparable perioperative outcomes. In the last decade, many authors faced up to the question whether TP or RP approach is better in performing $\mathrm{PN}$, especially with the diffusion of laparoscopic and robotic approaches. However, no definitive conclusions have been drawn and this seems to still remain a never-ending issue $[10-13,15]$.

One possible explanation could be that surgical approach is still mainly influenced by the surgeon's preference [22, 23]. In fact, even if the RP approach is burdened by the smaller working space and harder instruments handling, especially in conventional laparoscopy, many surgeons are more comfortable with such approach rather than TP in treating posterior tumors [22]. Conversely, some others underline the overcoming of these limitations with robotic technology, which augmented the surgeon's abilities to work in a small confined space [24, 25]. Moreover, even if the $\mathrm{RP}$ approach requires a strong knowledge of untraditional anatomic landmarks, it offers several advantages thanks to the direct access to the renal pedicle and avoidance of the bowel mobilization.

Another paramount issue is represented by the localization of renal tumor. Indeed, tumors localized in the posterior face or in the lateral margin or lower pole of the kidney can be easily approached with a RP access. Conversely, when a TP access is preferred, a prolonged and laborious, extensive isolation of kidney from adherent perinephric fat could be necessary. Tumors of the inferior pole can be easily managed with either TP or RP PN in most of the cases. Contrarily, some cases of renal tumors located on the anterior face of the kidney can be extremely challenging with a RP access, up to become extremely risky or almost impossible to manage if these lesions are close to the renal hilum.

In our study a propensity score matching was performed to select those cases with comparable patients' and tumor's characteristics. The number of patients treated with RP mi-PN for anterior tumors were low (37 cases, 9\%), while no case of peri-hilar tumor treated with RP mi-PN was reported. This introduces a selection bias and, again, it is mainly related to the surgeon's belief of an easier control over tumor resection and anatomical landmarks using a TP access. Despite the conclusions of this study cannot be generalized to all the types of renal tumors, to date, no randomized trials comparing TP and RP mi-PN are available. The current evidence come from both prospective and retrospective observational studies, single and multi-institutional matched pair cohorts and few review and meta-analyses [10-13, 15, 24].

Considered together, the results of the different studies are inconclusive, being affected by confounding factors and biases in the comparison of the cohorts of patients. For example, some studies considered different sample sizes, different preoperative patient- and tumor-related features or single centers experiences [26]. Moreover, in order to avoid potential limitations, other authors focused their attention only on specific surgical approaches, like laparoscopy or robotic surgery or on specific tumor features, like posterior or complex cases [27, 28]. In a recently published systematic review and meta-analysis comparing the RP and TP approaches for posteriorly located tumors treated with RAPN, Mclean et al. found no differences in terms of perioperative outcomes except for a lower operative time in RP PN [29]. They concluded that the most suitable approach for index patients' undergoing RAPN depends on surgeon expertise and familiarity with technique, considering the patient characteristics using a risk-stratified model.

Similar results were found by Xia et al. [30]. In their meta-analysis, they compared the perioperative outcomes of TP vs RP RAPN and showed no significant difference in terms of complication rate, conversion rate, WIT, EBL, and PSM rate. Only OT resulted marginally shorter in RP vs TP RAPN ( $p=0.05$, WMD: 28.03; 95\% CI 0.41-55.65). Other two meta-analyses comparing TP and RP LPN, including 7 and 8 studies respectively, showed that RP LPN had a shorter OT and hospital stay compared with TP RAPN [10, 11]. However, many of these studies concluded that RCTs and high-quality observational cohort studies with large sample size are needed to confirm their findings.

In this study, a large heterogeneous multi-institutional population was analyzed to be as much as possible representative of the real scenario and to reduce the biases related to single centers' or single surgeons' settings. A main limitation of this study was the absence of the assessment of each surgeon experience. Notwithstanding with these limitations, our results suggest that center experience could be identified as a main driver of the choice of the surgical access in mi-PN. In fact, RP access was more frequent in centers performing a higher number of PN/year and with a higher $\mathrm{PN} / \mathrm{RN}$ ratio per year compared to the TP group (56 vs 71 and $66.6 \%$ vs $74.0 \%, p<0.0001)$. These two surrogates can be representative of the experience of the surgeons within a center.

Concerning intraoperative variables, no differences were found in terms of EBL or ischemia time, confirming results also reported by other Institutions [12, 30]. Contrarily from other available studies [16, 24], we evaluated other surgical variables potentially influenced by the surgical approach, such as the renal pedicle management and the resection technique. No significant differences between TP and RP PN emerged in terms of clampless procedures ( $47.9 \%$ vs $51.6 \%$ ) and simple-enucleation rate ( $41.9 \%$ vs $40.9 \%)$.

Compared to most of the literature studies [13], we found a shorter median operative time in TP group (115 vs $150 \mathrm{~min}, p<0.0001)$. This result could be explained considering that the RP group is composed also by robotic 
procedures where the operative time was longer compared to laparoscopic RP PN. In fact, in the robotic approach, the creation of the virtual retroperitoneal space is performed laparoscopically to create a working space for robotic trocars: this could determine an increase of the overall operative time.

RP approach was associated to a slightly but statistically significant lower overall and surgical intraoperative complication rates (5\% vs $2.1 \%$ and $4.3 \%$ vs $1.7 \%, p=0.03$ and $p=0.04$, respectively). These evidences can be explained considering the advantages offered by RP approach in identifying easily the renal pedicle and avoiding direct contact with the intraperitoneal organs. In fact, stratifying the surgical intraoperative complications, higher number of vascular lesions and spleen injury were recorded in TP group ( 9 vs 4 and 2 vs 0 , respectively).

Focusing on postoperative outcomes, our findings are consistent with most of the current studies on this topic [10, $11,15,24]$. No differences were recorded in terms of postoperative complications, even stratifying according to ClavienDindo classification, as well as the PSM rate and functional outcomes. Patients managed with a RP access showed a shorter postoperative recovery, with and earlier drainage removal and discharge compared to those treated with TP PN. Similar results, explainable considering a smaller fluids drainage and a faster canalization in case of RP, were found in both pure laparoscopic and robotic case series [10, 15].

To the best of our knowledge this is the largest matched pair multi-institutional study comparing TP and RP in MI-PN setting. The strength of our results is to highlight the feasibility of both approaches, offering similar intra- and postoperative results. Indeed, this highlights that, if the surgeon is confident using either the RP or the TP access, he can modulate the choice more consciously, considering all the details of each single case. Notwithstanding its strengths, the study is not devoid of limitations. First, the design of the study was not randomized, and the access of each case was determined by surgeon choice and attitude. Even if the matching minimizes the potential biases related to the comparison of different populations, a selection bias related to the lower rate of anterior and peri-hilar renal tumors in the RP group could not be corrected. Thus, definitive conclusions on which surgical access should be preferred basing on tumor nephrometric characteristics cannot be drawn. Surgeon experience was not evaluated prospectively and for this reason it could not be added as parameter for the matching balance. On the other side, all the intraoperative characteristics, such as center volume, surgical approach and clamping, were not balanced a priori to provide a comprehensive representation of the surgical management of cases according to the baseline characteristics. Moreover, no detailed long-term functional data are available and postoperative oncological outcomes were not evaluated.

\section{Conclusions}

The results of this study suggest that both TP and RP are feasible approaches when performing MI-PN for cT1 tumors. Notwithstanding longer operative times, RP seems to have a slighter intraoperative complication rate with earlier postoperative recovery when compared with TP. Anyway, surgeons' preference and experience remain the tips of the balance in the decision towards the surgical access while performing a MI-PN.

Acknowledgements The RECORD 2 Project members: Vincenzo Altieri; Francesco Berardinelli; Antonio Celia; Elisabetta Costantini; Alberto Diminutto; Mario Falsaperla; Matteo Ferro; Maria Furlan; Gaetano Grosso; Alessandro Larcher; Vincenzo Li Marzi; Francesco Montorsi; Andrea Polara; Angelo Porreca; Riccardo Rizzetto; Marco Roscigno; Luigi Schips; Cesare Selli; Sergio Serni; Alchiede Simonato; Carlo Trombetta; Giuseppe Vespasiani; Alessandro Volpe.

Funding Open access funding provided by Università degli Studi di Firenze within the CRUI-CARE Agreement.

Open Access This article is licensed under a Creative Commons Attribution 4.0 International License, which permits use, sharing, adaptation, distribution and reproduction in any medium or format, as long as you give appropriate credit to the original author(s) and the source, provide a link to the Creative Commons licence, and indicate if changes were made. The images or other third party material in this article are included in the article's Creative Commons licence, unless indicated otherwise in a credit line to the material. If material is not included in the article's Creative Commons licence and your intended use is not permitted by statutory regulation or exceeds the permitted use, you will need to obtain permission directly from the copyright holder. To view a copy of this licence, visit http://creativecommons.org/licenses/by/4.0/.

\section{References}

1. Ljungberg B, Bensalah K, Canfield S, Dabestani S, Hofmann F, Hora $\mathrm{M}$ et al (2015) EAU guidelines for renal cell carcinoma: 2014 update. Eur Urol 67:913-924. https://doi.org/10.1016/j.eurur 0.2015.01.005

2. Ali S, Ahn T, Papa N, Perera M, Teloken P, Coughlin G et al (2019) Changing trends in surgical management of renal tumours from 2000 to 2016: a nationwide study of Medicare claims data. ANZ J Surg. https://doi.org/10.1111/ans.15385

3. Minervini A, Rosaria Raspollini M, Tuccio A, Di Cristofano C, Siena G, Salvi M et al (2014) Pathological characteristics and prognostic effect of peritumoral capsule penetration in renal cell carcinoma after tumor enucleation. Urol Oncol 32(50):e15-22. https://doi.org/10.1016/j.urolonc.2013.07.018

4. Li J, Zhang Y, Teng Z, Han Z (2019) Partial nephrectomy versus radical nephrectomy for cT2 or greater renal tumors: a systematic review and meta-analysis. Minerva Urol Nefrol 71:435-444. https ://doi.org/10.23736/S0393-2249.19.03470-2

5. Larcher A, Capitanio U, Terrone C, Volpe A, De Angelis P, Deho $F$ et al (2016) Elective nephron sparing surgery decreases other cause mortality relative to radical nephrectomy only in specific subgroups of patients with renal cell carcinoma. J Urol 196:10081013. https://doi.org/10.1016/j.juro.2016.04.093 
6. Capitanio U, Larcher A, Terrone C, Antonelli A, Volpe A, Fiori C et al (2016) End-stage renal disease after renal surgery in patients with normal preoperative kidney function: balancing surgical strategy and individual disorders at baseline. Eur Urol. https:// doi.org/10.1016/j.eururo.2016.03.023

7. Veccia A, Dell'oglio P, Antonelli A, Minervini A, Simone G, Challacombe B et al (2019) Robotic partial nephrectomy versus radical nephrectomy in elderly patients with large renal masses. Minerva Urol Nefrol. https://doi.org/10.23736/S0393 -2249.19.03583-5

8. Serni S, Vittori G, Frizzi J, Mari A, Siena G, Lapini A et al (2015) Simple enucleation for the treatment of highly complex renal tumors: perioperative, functional and oncological results. Eur J Surg Oncol. https://doi.org/10.1016/j.ejso.2015.02.019

9. Mari A, Campi R, Schiavina R, Amparore D, Antonelli A, Artibani W et al (2019) Nomogram for predicting the likelihood of postoperative surgical complications in patients treated with partial nephrectomy : a prospective multicentre observational study (the RECORd 2 project). BJU Int. https://doi.org/10.1111/ bju. 14680

10. Fan X, Xu K, Lin T, Liu H, Yin Z, Dong W et al (2013) Comparison of transperitoneal and retroperitoneal laparoscopic nephrectomy for renal cell carcinoma: a systematic review and meta-analysis. BJU Int 111:611-621. https://doi.org/10.1111/ j.1464-410X.2012.11598.x

11. Ren T, Liu Y, Zhao X, Ni S, Zhang C, Guo C et al (2014) Transperitoneal approach versus retroperitoneal approach: a meta-analysis of laparoscopic partial nephrectomy for renal cell carcinoma. PLoS ONE 9:e91978. https://doi.org/10.1371/journal.pone.00919 78

12. Ng CS, Gill IS, Ramani AP, Steinberg AP, Spaliviero M, Abreu SC et al (2005) Transperitoneal versus retroperitoneal laparoscopic partial nephrectomy: patient selection and perioperative outcomes. J Urol 174:846-849. https://doi.org/10.1097/01. ju.0000169259.49754.02

$13 \mathrm{Fu}$ J, Ye S, Ye H (2015) Retroperitoneal versus transperitoneal laparoscopic partial nephrectomy: a systematic review and metaanalysis. Chin Med Sci J Chung-Kuo i Hsueh k'o Hsueh Tsa Chih 30:239-244. https://doi.org/10.1016/s1001-9294(16)30007-4

14. Porpiglia F, Mari A, Bertolo R, Antonelli A, Bianchi G, Fidanza $F$ et al (2016) Partial nephrectomy in clinical T1b renal tumors: multicenter comparative study of open, laparoscopic and robotassisted approach (the RECORd Project). Urology 89:45-51. https ://doi.org/10.1016/j.urology.2015.08.049

15. Arora S, Heulitt G, Menon M, Jeong W, Ahlawat RK, Capitanio U et al (2018) Retroperitoneal vs transperitoneal robot-assisted partial nephrectomy: comparison in a multi-institutional setting. Urology 120:131-137. https://doi.org/10.1016/j.urolo gy.2018.06.026

16. Schiavina R, Mari A, Antonelli A, Bertolo R, Bianchi G, Borghesi $M$ et al (2015) A snapshot of nephron-sparing surgery in Italy: a prospective, multicenter report on clinical and perioperative outcomes (the RECORd 1 project). Eur J Surg Oncol. https://doi. org/10.1016/j.ejso.2014.12.001

17. Austin PC (2011) An introduction to propensity score methods for reducing the effects of confounding in observational studies. Multivariate Behav Res 46:399-424. https://doi.org/10.1080/00273 171.2011.568786

18. Mari A, Antonelli A, Bertolo R, Bianchi G, Borghesi M, Ficarra $\mathrm{V}$ et al (2017) Predictive factors of overall and major postoperative complications after partial nephrectomy: results from a multicenter prospective study (The RECORd 1 project). Eur J Surg Oncol 43:823-830. https://doi.org/10.1016/j.ejso.2016.10.016

19. Minervini A, Siena G, Antonelli A, Bianchi G, Bocciardi AM, Cosciani Cunico S et al (2014) Open versus laparoscopic partial nephrectomy for clinical T1a renal masses: a matched-pair comparison of 280 patients with TRIFECTA outcomes (RECORd Project). World J Urol. https://doi.org/10.1007/s00345-013-1155-7

20. Grivas N, Kalampokis N, Larcher A, Tyritzis S, Rha KH, Ficarra $\mathrm{V}$ et al (2019) Robot-assisted versus open partial nephrectomy: comparison of outcomes. A systematic review. Minerva Urol Nefrol 71:113-120. https://doi.org/10.23736/S0393-2249.19.03391-5

21. Bravi CA, Larcher A, Capitanio U, Mari A, Antonelli A, Artibani W et al (2019) Perioperative outcomes of open, laparoscopic, and robotic partial nephrectomy: a prospective multicenter observational study (The RECORd 2 Project). Eur Urol Focus. https://doi. org/10.1016/j.euf.2019.10.013

22. Desai MM, Strzempkowski B, Matin SF, Steinberg AP, Ng C, Meraney AM et al (2005) Prospective randomized comparison of transperitoneal versus retroperitoneal laparoscopic radical nephrectomy. J Urol 173:38-41. https://doi.org/10.1097/01. ju.0000145886.26719.73

23. Khandwala YS, Jeong IG, Han DH, Kim JH, Li S, Wang Y et al (2017) Surgeon preference of surgical approach for partial nephrectomy in patients with baseline chronic kidney disease: a nationwide population-based analysis in the USA. Int Urol Nephrol 49:1921-1927. https://doi.org/10.1007/s11255-017-1688-6

24. Gin GE, Maschino AC, Spaliviero M, Vertosick EA, Bernstein ML, Coleman JA (2014) Comparison of perioperative outcomes of retroperitoneal and transperitoneal minimally invasive partial nephrectomy after adjusting for tumor complexity. Urology 84:1355-1360. https://doi.org/10.1016/j.urology.2014.07.045

25. Pavan N, Derweesh I, Hampton LJ, White WM, Porter J, Challacombe BJ et al (2018) Retroperitoneal robotic partial nephrectomy: systematic review and cumulative analysis of comparative outcomes. J Endourol 32:591-596. https://doi.org/10.1089/ end.2018.0211

26. Laviana AA, Tan H-J, Hu JC, Weizer AZ, Chang SS, Barocas DA (2018) Retroperitoneal versus transperitoneal robotic-assisted laparoscopic partial nephrectomy: a matched-pair, bicenter analysis with cost comparison using time-driven activity-based costing. Curr Opin Urol 28:108-114. https://doi.org/10.1097/MOU.00000 00000000483

27. Paulucci DJ, Beksac AT, Porter J, Abaza R, Eun DD, Bhandari A et al (2019) A multi-institutional propensity score matched comparison of transperitoneal and retroperitoneal partial nephrectomy for cT1 posterior tumors. J Laparoendosc Adv Surg Tech A 29:29-34. https://doi.org/10.1089/lap.2018.0313

28. Choi C, Kang M, Sung HH, Jeon HG, Jeong BC, Jeon SS et al (2019) Comparison by pentafecta criteria of transperitoneal and retroperitoneal robotic partial nephrectomy for large renal tumors. J Endourol. https://doi.org/10.1089/end.2019.0410

29 McLean A, Mukherjee A, Phukan C, Veeratterapillay R, Soomro N, Somani B et al (2019) Trans-peritoneal vs. retroperitoneal robotic assisted partial nephrectomy in posterior renal tumours: need for a risk-stratified patient individualised approach. A systematic review and meta-analysis. J Robot Surg. https://doi. org/10.1007/s11701-019-00973-8

30. Xia L, Zhang X, Wang X, Xu T, Qin L, Zhang X et al (2016) Transperitoneal versus retroperitoneal robot-assisted partial nephrectomy: a systematic review and meta-analysis. Int J Surg 30:109-115. https://doi.org/10.1016/j.ijsu.2016.04.023

Publisher's Note Springer Nature remains neutral with regard to jurisdictional claims in published maps and institutional affiliations. 


\section{Affiliations}

Francesco Porpiglia ${ }^{1} \cdot$ Andrea Mari $^{2}$. Daniele Amparore ${ }^{1} \cdot$ Cristian Fiori $^{1} \cdot$ Alessandro Antonelli $^{3} \cdot$ Walter Artibani $^{4}$. Pierluigi Bove ${ }^{5}$. Eugenio Brunocilla ${ }^{6,7}$. Umberto Capitanio ${ }^{8}$. Luigi Da Pozzo ${ }^{9}$. Fabrizio Di Maida ${ }^{1}$ Paolo Gontero ${ }^{10}$. Nicola Longo ${ }^{11}$. Giancarlo Marra ${ }^{10}$ - Bernardo Rocco ${ }^{12,13}$. Riccardo Schiavina ${ }^{6}$. Claudio Simeone ${ }^{3}$. Salvatore Siracusano ${ }^{4} \cdot$ Riccardo Tellini $^{1} \cdot$ Carlo Terrone $^{14}$. Donata Villari ${ }^{15} \cdot$ Vincenzo Ficarra $^{16}$ - Marco Carini ${ }^{1}$. Andrea Minervini ${ }^{1,17}$. The RECORD 2 Project

1 Division of Urology, Department of Oncology- School of Medicine, University of Turin, San Luigi Hospital, Orbassano, Turin, Italy

2 Department of Urology, Unit of Oncologic Minimally-Invasive Urology and Andrology, Careggi Hospital, University of Florence, Florence, Italy

3 Department of Urology, Spedali Civili Hospital, University of Brescia, Brescia, Italy

4 Department of Urology, Azienda Ospedaliera Universitaria Integrata (A.O.U.I.), Verona, Italy

5 Department of Urology, University Hospital of Tor Vergata, Rome, Italy

6 Department of Urology, University of Bologna, Bologna, Italy

7 Department of Experimental, Diagnostic, and Specialty Medicine, University of Bologna, Bologna, Italy

8 Unit of Urology, Division of Experimental Oncology, URI-Urological Research Institute, Vita-Salute San Raffaele University, IRCCS San Raffaele Scientific Institute, Milan, Italy

9 Department of Urology, Papa Giovanni XXIII Hospital, Bergamo, Italy
10 Division of Urology, Department of Surgical Sciences, San Giovanni Battista Hospital, University of Studies of Torino, Turin, Italy

11 Department of Urology, University Federico II of Naples, Naples, Italy

12 Department of Urology, Fondazione IRCCS Ca' Granda, Ospedale Maggiore Policlinico, Policlinico, University of Milan, Milan, Italy

13 Department of Urology, University of Modena and Reggio Emilia, Modena, Italy

14 Department of Urology, University of Genova, Genova, Italy

15 Department of Urology, Unit of Urological Minimally Invasive Robotic Surgery and Renal Transplantation, Careggi Hospital, University of Florence, Florence, Italy

16 Department of Human and Paediatric Pathology, Gaetano Barresi, Urologic Section, University of Messina, Messina, Italy

17 Department of Urology, Careggi Hospital, San Luca Nuovo, University of Florence, Florence, Italy 